\section{Microcirculation at Different Parts of the Foot in Healthy Subjects}

\author{
Stefan Rosfors* \\ Department of Clinical Science and Education, Section of Clinical Physiol- \\ ogy, Karolinska Institutet, Södersjukhuset, Stockholm, Sweden
}

\begin{abstract}
The study aimed to describe microcirculation at different parts of the foot with two different methods in healthy controls. Furthermore, the effect of oxygen inhalation and the relationship between results with these two methods were evaluated.

Methods: Microcirculation of the foot was evaluated on 15 healthy subjects (aged 22-37 years) by two methods: Transcutaneous Oxygen Pressure (TcPO2) with and without oxygen provocation and Laser Doppler Flux (LDF) with heat provocation. Four different measuring sites were used: one distal and one proximal point at the dorsal foot (the distal is the standard position), one point at the medial plantar part and one at the anterolateral part of the foot. The measuring points were distributed on parts of the foot supplied by three different end-arteries (so called angiosome).

Results: TcPO2 levels were significantly higher at all three measuring points than at the standard position at the distal dorsal foot. Median TcPO2 was $68 \mathrm{~mm} \mathrm{Hg}$ at the standard position compared with 76 $\mathrm{mm} \mathrm{Hg}$ at the dorsal proximal, $73 \mathrm{~mm} \mathrm{Hg}$ at the medial and $83 \mathrm{~mm}$ $\mathrm{Hg}$ at the anterolateral electrode position. After oxygen provocation only the proximal dorsal point remained significantly higher than the standard position. Heat-provoked LDF did not differ between different parts of the foot with an increase of more than $400 \%$ seen at all probe positions in all subjects. Oxygen inhalation increased TcPO2 to median levels around $200 \mathrm{~mm} \mathrm{Hg}$, without affecting LDF. Both TCPO2 and LDF results were unrelated to different angiosome.

Conclusion: In healthy subjects TcPO2 levels are slightly higher at proximal than at distal parts of the foot, but LDF did not differ between different parts of the foot. Although both methods are used to study the microcirculation there are no correlations between TcPO2 and LDF, which reflect that these methods use different mechanisms and different measurement principles.

Keywords: Foot circulation; Laser doppler flowmetry; Regional blood flow; Skin circulation; Transcutaneous oximetry
\end{abstract}

*Corresponding author: Stefan Rosfors, Department of Clinical Science and Education, Section of Clinical Physiology, Karolinska Institutet, Södersjukhuset, Stockholm, Sweden, Tel: +46 86163535; E-mail: stefan.rosfors@sodersjukhuset.se

Citation: Rosfors S (2016) Microcirculation at Different Parts of the Foot in Healthy Subjects. J Non invasive Vasc Invest 1: 005.

Received: November 9, 2016; Accepted: December 29, 2016; Published: December 31, 2016

\section{Introduction}

The microcirculation of the skin is complex with a number of regulating and modulating systems, nevertheless its importance for tissue viability cannot be over emphasized [1]. The microcirculation of the foot can be assessed with Transcutaneous Oxygen Pressure measurements (TcPO2) and with Laser Doppler Flux (LDF), and for example both methods have been used to predict ulcer healing [2,3]. However, little is known regarding how these two methods compare to each other, and regarding the effect of different positioning of electrodes/ probes.

Local microcirculation of the foot can be described in terms of the angiosome model, a concept that has gained in interest during the last decade [4]. Basically it refers to a block of tissue perfusion by a specific end-artery. At the foot the three main arteries give rise to six end-arteries, each supplying an angiosome; three from the posterior tibial artery, two from the peroneal artery and one from the anterior tibial artery [4]. The angiosome concept of the foot has received special attention regarding choice of target artery for revascularization in order to achieve best ulcer healing.

The aim of this study is to investigate the microcirculation of the foot on healthy subjects with two different methods (TcPO2 and heat-provoked LDF) in order to describe regional differences in parts of the foot supplied by different end-arteries, and to study relationships between results obtained with these two methods.

\section{Material and Methods}

\section{Healthy subjects}

Fifteen healthy subjects were included; 6 males and 9 females with a median age of 28 years (range 22 to 37 years). Inclusion criteria were: age between 18 and 40 years, no present or previous smoking, no diabetes, no lower limb symptoms, ulcerations or other skin changes.

\section{Positioning of electrodes/probes and experimental set-up}

The standard position used in most previous studies on microcirculation is the dorsum of the foot in the intermetatarsal space proximal to the first and second toe. This part of the foot belongs to the dorsalis pedis angiosome, with blood supply from the dorsalis pedis artery (from the anterior tibial artery). For comparison three other positions were chosed: one from the same angiosome but with a more proximal location, and two from other parts of the foot representing other angiosome and with blood supply emanating from the other two main arteries of the lower limb. This set-up allowed a comparison of results from measuring sites within the same angiosome, as well as from other angiosome with different supplying arteries.

- Dorsal distal: The distal part of dorsalis pedis angiosome (supplied by the anterior tibial artery), standard position

- Dorsal proximal: The proximal part of the dorsalis pedis angiosome (supplied by the anterior tibial artery)

- Medial: The medial plantar artery angiosome (supplied by the posterior tibial artery) 
- Anterolateral: The anterior perforating branch angiosome (supplied by the peroneal artery)

These investigations were made in two steps; first positions 1 and 2 and after replacement of the electrodes/probes positions 3 and 4 were investigated (Figure 1). The patients were studied in supine position. First two LD probes were attached and baseline values were sampled followed by activation of the built-in heater system. The calibrated TcPO2 electrodes were then attached close to the LD probes, heating of the TcPO2 electrodes was started and then sampling for both TcPO2 and LDF, followed by oxygen provocation. Only one limb per patient was studied (randomly 7 right limbs and 8 left limbs).

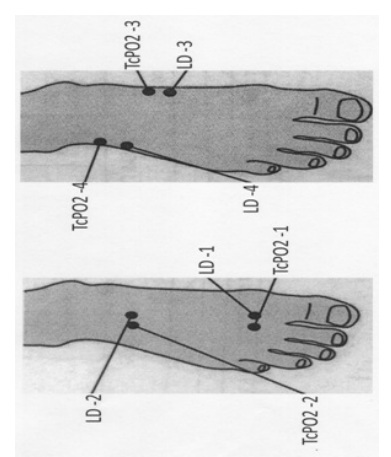

Figure 1: Positioning for measurements of Transcutaneous Oxygen Pressure (TcPO2) and LD (Laser Doppler) and corresponding angiosome.

1. Dorsal distal: The distal part of dorsalis pedis angiosome, (supplied by the anterior tibial artery), standard position

2. Dorsal proximal: The proximal part of the dorsalis pedis angiosome (supplied by the anterior tibial artery)

3. Medial: The medial plantar artery angiosome (supplied by the posterior tibial artery).

4. Anterolateral: The anterior perforating branch angiosome (supplied by the peroneal artery)

\section{Transcutaneous Oxygen Pressure (TcPO2)}

The two TcPO2 electrodes (Periflux System 5040, Perimed, Järfälla, Sweden) were calibrated for 3 minutes and then electronically heated to 45 degrees C. Continuous measurement of $\mathrm{TcPO} 2$ was performed for 15 minutes and the average of the last minute was recorded. Then the subjects breathed $100 \%$ oxygen during 10 minutes through a closed mask system and the highest TcPO2-levels at end of oxygen breathing were noted.

\section{Laser Doppler Flux (LDF)}

The two combined heat and LD probes (Periflux System 5040, Perimed, Järfälla, Sweden) were attached to the skin avoiding bony areas and superficial vessels. A baseline level was sampled for 3 minutes before the electronically heating was started by the built-in heater system. Heat-provoked LDF was registered as the mean of 1 minute recording after approximately 16-18 minutes, although in general a new stable level was reached within 10 minutes. The LD probes were left in place during the subsequent 10 minute period of TcPO2 oxygen provocation, allowing detection of any possible change in LDF during oxygen inhalation.

\section{Statistics and ethics}

Values are presented as medians and ranges/quartiles. Differences were tested for significance using Wilcoxon's signed rank test. Spearman's rank order correlations were used to study relations between TcPO2 and LD variables (Statistica 12, StatSoft Inc., Tulsa, OK, USA). Statistical significance was assumed at $\mathrm{P}<0.05$.
The study was approved by the local ethics committee and conducted according to the declaration of Helsinki. Written informed consent was obtained from all participants.

\section{Results}

\section{TcPO2}

TcPO2 values were significantly higher at electrode positions 2,3 and 4 compared to the standard position ( 1 versus $2, \mathrm{P}<0.05 ; 1$ versus $3, \mathrm{P}<0.05 ; 1$ versus $4, \mathrm{P}<0.01$ ). Oxygen breathing induced a considerable rise in $\mathrm{TcPO} 2$ on all electrode positions. After oxygen breathing there remained a significant difference only between the dorsal distal (reference point) and the dorsal proximal (position 2) $(\mathrm{P}<0.05)$. In general, $\mathrm{TcPO} 2$ was above $60 \mathrm{~mm} \mathrm{Hg}$ at all positions and increased to values above $100 \mathrm{~mm} \mathrm{Hg}$ after oxygen breathing. At the standard position only, a few subjects had lower values than 60 and $100 \mathrm{~mm}$ $\mathrm{Hg}$, respectively (Table 1). The results did not differ between males and females.

\begin{tabular}{|c|c|c|c|}
\hline & Median & Range & Lower-upper quartile \\
\hline \multicolumn{4}{|l|}{ TcPO2 (mm Hg) } \\
\hline Dorsal distal & 68 & $54-88$ & $60-78$ \\
\hline Dorsal proximal & 76 & $63-91$ & $72-81$ \\
\hline Medial & 73 & $58-89$ & $71-83$ \\
\hline Anterolateral & 83 & $69-103$ & $77-94$ \\
\hline \multicolumn{4}{|c|}{ TcPO2 breathing oxygen } \\
\hline Dorsal distal & 209 & $68-341$ & $106-247$ \\
\hline Dorsal proximal & 246 & $122-322$ & 169-302 \\
\hline Medial & 190 & $104-277$ & $138-235$ \\
\hline Anterolateral & 221 & $107-289$ & $151-268$ \\
\hline \multicolumn{4}{|c|}{ Heat-provoked LDF (PU) } \\
\hline Dorsal distal & 182 & $62-366$ & $137-226$ \\
\hline Dorsal proximal & 170 & $84-393$ & $122-227$ \\
\hline Medial & 161 & $66-385$ & $96-174$ \\
\hline Anterolateral & 230 & $76-407$ & $159-262$ \\
\hline
\end{tabular}

Table 1: TcPO2 in $\mathrm{mm} \mathrm{Hg}$ and heat-provoked Laser Doppler Flux (LDF) in Perfusion Units (PU) at 4 different sites on the foot in 15 healthy subjects.

\section{LDF}

The baseline level was low $(<20 \mathrm{PU})$ and increased more than $400 \%$ with heating in all subjects at all measuring points. LDF after heating varied substantially and at the different measuring point's values between 62 and 393 could be found (Table 1). There was no significant difference in heat-provoked LDF between the standard position and the other probe positions. The oxygen breathing did not induce any additional change in LDF at any of the probe positions. At the anterolateral position males had slightly higher heat-provoked LDF than females $(P<0.05)$, otherwise there were no gender differences. In general, LDF results did not correlate with those obtained with TcPO2. Only at the medial measuring point (probe position 3 ) there was a significant relation between $\mathrm{TcPO} 2$ and heat-provoked $\mathrm{LDF}$ ( $\mathrm{rho}=0.62, \mathrm{P}$ $<0.05)$.

\section{Discussion}

In the present study TcPO2 and LDF were used simultaneously to assess microcirculation of the foot in healthy subjects with electrodes/ probes placed on four different points distributed on three angiosome. TcPO2 results, but not those obtained with LDF, differed significantly 
between proximal and distal measuring positions of the foot. These differences seen with TcPO2 were unrelated to angiosome of the foot.

For TcPO2 it is common to use a fixed electrode position at the dorsum of the foot in the first intermetatarsal space just proximal to the first and second toe (denoted standard position in the present study) [5]. Here a level below $30 \mathrm{~mm} \mathrm{Hg}$ is proposed to denote critical limb ischemia [1]. Different positions in the vicinity of problem wounds are sometimes used, for example to select patients that are suitable for hyperbaric oxygen therapy [6]. Moreover, the electrodes can be applied at different levels of the foot and the lower limb to assess the proper level for amputation [7]. The present results demonstrated systematically higher levels at the proximal foot (dorsal, medial and anterolateral parts) than at the distal foot. This difference was not related to angiosome, since it was a significant difference between electrode positions also within the same angiosome (i.e., higher levels at the proximal dorsal foot than at the distal dorsal). This is not surprising, on the contrary rather expected since angiosome are supposed to be of importance in patients with compromised circulation and diseased arterial interconnections $[4,8]$. Thus, in normal subjects there are small, but significant, regional differences in microcirculation assessed with TcPO2. These differences are difficult to explain, but anatomic variations in healthy subjects might play a role.

When the six angiosome of the foot were described, an interest emerged regarding revascularization of patients with critical ischemia and ulcers/gangrene [9]. Some early results suggested a better outcome in terms of wound healing when the procedure was directed towards the vessel feeding the angiosome containing the ulcer (DR- Direct Revascularization), compared to when the procedure included only vessels not feeding the affected angiosome directly (IR- Indirect Revascularisation) [10]. However, in larger series and in reviews the results were much more divergent and the role of the angiosome concept in revascularization of ischemic limbs remains to some extent unclear [11]. For example it has been suggested that the concept has higher relevance regarding ulcer healing in diabetic patients with known poor collateralization compared to non-diabetics [12]. In recent study Rother et al., used microcirculatory methods with angiosome-related measuring points to study the effect of DR and IR in patients with critical limb ischemia [13]. Their results could not support an angiosome concept of the foot regarding changes in microperfusion.

In general the healthy subjects in the present study had basal TcPO2 levels between 60 and $80 \mathrm{~mm} \mathrm{Hg}$ that increased to $150-200 \mathrm{~mm} \mathrm{Hg}$ after oxygen inhalation. The low basal LDF ( $<20 \mathrm{PU})$ increased with heat provocation to around 180-200 PU. Oxygen inhalation did not further affect LDF, an expected finding although not earlier described. According to an earlier suggested classification system for interpreting TcPO2 and LDF all healthy controls except one could be denoted as normal [3]. The exception was a 29 -year old female with $402 \%$ increase in heat-provoked LDF at the standard position and $426 \%$ increase at the proximal dorsal probe (normal $>500 \%$ ). We consider this to be interpreted as within the normal variability range, rather than to implicate microvascular disease.

In the present study apparently healthy subjects were studied. One possible limitation was that no standard methods were used to prove "normality". In order to control for that only asymptomatic subjects below 40 years without risk factors were chosen. The fact that all the subjects were found to have $\mathrm{TcPO} 2$ within the normal range at all measuring points at the foot strongly supports that the subjects were free of both macro- and microvascular disease. Most authors agree that a normal TcPO2 can be used to rule out both macro- and microvascular disease, and $50 \mathrm{~mm} \mathrm{Hg}$ at foot level is a generally found lower limit of normality [2,14-18]. Another possible limitation was the rather small number of only 15 subjects included. A larger sample would have increased the usefulness of the present study, since then also results regarding differences between age groups could have been assessed and possibly also reference values for various microcirculatory variables.

There was in general no significant relation between $\mathrm{TcPO} 2$ values and LDF. This is not surprising since two different measuring principles are used; TcPO2 with measure of oxygen in skin capillaries and LD with assessment of tissue reserve capacity recruiting response from somewhat more deeply located structures which besides capillaries also can include arterioles and venules [3]. TcPO2 and LDF were chosen since they are commonly used in studies of microvascular function, but with limited knowledge regarding relationship between results obtained with these two methods.

\section{Conclusion}

Studies of microcirculation at different parts of the foot in healthy controls show TcPO2 levels at $60-80 \mathrm{~mm} \mathrm{Hg}$, slightly higher at proximal than at distal parts of the foot. The results from different parts were not related to different angiosome. Heat-provoked LDF did not differ between different parts of the foot with an increase of more than $400 \%$ seen at all electrode positions in all subjects. Oxygen inhalation increased TcPO2 levels to more than $100 \mathrm{~mm} \mathrm{Hg}$, without affecting LDF. In general, there were no correlations between TcPO2 values and LDF due to different mechanisms and different measurement principles. The present results provide increased knowledge of regional microcirculation and of methods used to study microvascular function.

\section{Acknowledgement}

The skilful contribution of technologist Malin Lindholm to all microcirculatory studies is gratefully acknowledged.

\section{References}

1. Norgren L, Hiatt WR, Dormandy JA, Nehler MR, Harris KA, et al. (2007) Inter-Society Consensus for the Management of Peripheral Arterial Disease (TASC II). J Vasc Surg 45: 5-67.

2. Sheffield PJ, Buckley CJ (2004) Transcutaneous oximetry. In: Paul J Sheffield, Caroline E Fife, Adrianne PS Smith (eds.). Wound Care Practice. Best Publishing, Flagstaff Arizona, USA. Pg no: 117-136.

3. Sheffield PJ, Buckley CJ (2004) Laser Doppler flowmetry. In: Paul J Sheffield Caroline E Fife, Adriane PS Smith (eds.) Wound Care Practice. Flagstaff, AZ: Best Publishing, Flagstaff Arizona, USA. Pg no: 137-156.

4. Houlind K, Christensen J (2013) The role of the angiosome model in treatment of critical limb ischemia. In: Wilbert S Aronow (ed.). Artery Bypass. INTECH Open Access Publisher, Croatia. Pg no: 426-434.

5. Rosfors S, Kanni L, Nyström T (2016) The impact of transcutaneous oxygen pressure measurement in patients with suspected critical lower limb ischemia. Int Angiol 35: 492-497.

6. Niinikoski JH (2004) Clinical hyperbaric oxygen therapy, wound perfusion, and transcutaneous oximetry. World J Surg 28: 307-311.

7. Sarin S, Shami S, Shields DA, Scurr JH, Smith PD (1991) Selection of amputation level: a review. Eur J Vasc Surg 5: 611-620.

8. Clemens MW, Attinger CE (2010) Angiosomes and wound care in the diabetic foot. Foot Ankle Clin 15: 439-464.

9. Attinger CE, Evans KK, Bulan E, Blume P, Cooper P (2006) Angiosomes of the foot and ankle and clinical implications for limb salvage: reconstruction, incisions, and revascularization. Plast Reconstr Surg 117: 261-293. 
10. Neville RF, Attinger CE, Bulan EJ, Ducic I, Thomassen M, et al. (2009) Revascularization of a specific angiosome for limb salvage: does the target artery matter? Ann Vasc Surg 23: 367-373.

11. Sumpio BE, Forsythe RO, Ziegler KR, van Baal JG, Lepantalo MJ, et al. (2013) Clinical implications of the angiosome model in peripheral vascular disease. J Vasc Surg 58: 814-826.

12. Georgakarakos E, Papanas N, Papadaki E, Georgiadis GS, Maltezos E, et al. (2013) Endovascular treatment of critical ischemia in the diabetic foot: new thresholds, new anatomies. Angiology 64: 583-591.

13. Rother U, Kapust J, Lang W, Horch RE, Gefeller O, et al. (2015) The Angiosome Concept Evaluated on the Basis of Microperfusion in Critical Limb Ischemia Patients-an Oxygen to See Guided Study. Microcirculation 22: 737-743.

14. Byrne P, Provan JL, Ameli FM, Jones DP (1984) The use of transcutaneous oxygen tension measurements in the diagnosis of peripheral vascular insufficiency. Ann Surg 200: 159-165.
15. Mannarino E, Maragoni G, Pasqualini L, Sanchini R, Rossi P, et al. (1987) Transcutaneous oxygen tension behavior in the different stages of peripheral vascular disease and its correlation with ankle/arm pressure ratio and calf blood flow. Angiology 38: 463-468.

16. Eickhoff JH, Engell HC (1981) Trancutaneous oxygen tension (tcPO2) measurements on the foot in normal subjects and in patients with peripheral arterial disease admitted for vascular surgery. Scand J Clin Lab Invest 41: 743-748.

17. Rooke TW (1992) The use of transcutaneous oximetry in the noninvasive vascular laboratory. Int Angiol 11: 36-40.

18. Sheffield PJ (1998) Measuring tissue oxygen tension: a review. Undersea Hyperb Med 25: 179-188. 